\title{
Optimal channel decision of retailers in the dual-channel supply chain considering consumer preference for delivery lead time
}

\author{
Hu, Y.S. ${ }^{a, b}$, Zeng, L.H. ${ }^{a}$, Huang, Z.L. ${ }^{c}$, Cheng, Q. ${ }^{\text {c, }}$

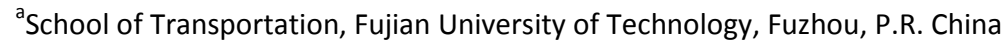 \\ ${ }^{\mathrm{b}} \mathrm{S}$ chool of Business, Maynooth University, Maynooth, County Kildare, Republic of Ireland \\ ${ }^{c}$ Research Center of Logistics, Fuzhou University, Fuzhou, P.R. China
}

\section{ABSTRACT}

Facing competition from manufacturers' online direct channels, how retailers make sales channel decisions to increase consumer stickiness has become the core concern of the industry and academia. Empirical research showed that delivery lead time is a key factor that affects consumers' preference for online channels. To analyze the impact of consumer delivery time preference on channel selection and pricing strategy of retailers, consumer delivery lead time preference function was improved from a linear function to an exponential function and consumer demand under the mixed dual-channel supply chain of manufacturer and retailer was derived. Then, the Stackelberg game models under different channel strategies of retailer were established and solved. Results show that consumer preference for delivery lead time has four implications on the channel decision of retailers under manufacturer encroachment in the dual-channel supply chain. First, the dual retail channels strategy is the optimal choice for retailers, and the profit margins that a retailer obtains from dual retail channels supply chain and single online retail channel supply chain will increase as consumers' delivery lead time preference coefficient increases. Second, the optimal pricing of online retail channel and offline retail channel is positively related to consumers' delivery lead time preference coefficient. By contrast, the optimal pricing of online direct channel is negatively related to consumers' delivery lead time preference coefficient. Third, the optimal pricing of online retail channel is higher than that of offline retail and online direct channels. Fourth, a retailer and a manufacturer can adopt a compensation-based whole price contract to address the conflict brought about by the optimal channel choice of the retailer. This study introduces consumer delivery lead time preference into retailer channel decision making and provides a theoretical reference for retailer's mixed channel construction in practice.
\end{abstract}

\author{
ARTICLE INFO \\ Keywords: \\ Supply chain; \\ Dual-channel; \\ Consumer preference; \\ Delivery lead time preference; \\ Channel selection; \\ Channel coordination \\ *Corresponding author: \\ chengquan@fzu.edu.cn \\ (Cheng, Q.) \\ Article history: \\ Received 20 September 2020 \\ Revised 6 December 2020 \\ Accepted 9 December 2020
}

\section{References}

[1] Chiang, W.Y.K., Monahan, G.E. (2005). Managing inventories in a two-echelon dual-channel supply chain, European Journal of Operational Research, Vol. 162, No. 2, 325-341, doi: 10.1016/j.ejor.2003.08.062.

[2] Hu, H., Wu, Q., Han, S., Zhang, Z. (2020). Coordination of dual-channel supply chain with perfect product considering sales effort, Advances in Production Engineering \& Management, Vol. 15, No. 2, 192-203, doi: 10.14743/ apem2020.2.358.

[3] Tsay, A.A., Agrawal, N. (2004). Channel conflict and coordination in the E-commerce age, Production and Operations Management, Vol. 13, No. 1, 93-110, doi: 10.1111/j.1937-5956.2004.tb00147.x. 
[4] Edinger-Schons, L.M., Lengler-Graiff, L., Scheidler, S., Mende, G., Wieseke, J. (2020). Listen to the voice of the customer - First steps towards stakeholder democracy, Business Ethics: A European Review, Vol. 29, No. 3, 510527, doi:10.1111/beer.12252.

[5] Hu, Y.S., Lin, J., Su, X.-L. (2020). Channel selection decision in a dual-channel supply chain: A consumer driven perspective, IEEE Access, Vol. 8, 145634 -145648, doi: 10.1109/ACCESS.2020.3014396.

[6] Jian, M., Wang, Y.L. (2018). Decision-making strategies in supply chain management with a waste averse and stockout averse manufacturer, Advances in Production Engineering \& Management, Vol. 13, No. 3, 345-357, doi: 10.14743/apem2018.3.295.

[7] Devaraj, S., Fan, M., Kohli, R. (2002). Antecedents of B2C channel satisfaction and preference: Validating ecommerce metrics, Information Systems Research, Vol. 13, No. 3, 316-333, doi: 10.1287/isre.13.3.316.77.

[8] Rohm, A.J., Swaminathan, V. (2004). A typology of online shoppers based on shopping motivations, Journal of Business Research, Vol. 57, No. 7, 748-757, doi: 10.1016/S0148-2963(02)00351-X.

[9] Gupta, A., Su, B.-C., Walter, Z. (2004). An empirical study of consumer switching from traditional to electronic channels: A purchase-decision process perspective, International Journal of Electronic Commerce, Vol. 8, No. 3, 131-161, doi: 10.1080/10864415.2004.11044302.

[10] Buntak, K., Kovačić, M., Mutavdžija, M. (2019). Internet of things and smart warehouses as the future of logistics, Tehnicki Glasnik - Technical Journal, Vol. 13, No. 3, 248-253, doi: 10.31803/tg-20190215200430.

[11] Gallego-Gómez, C., de-Pablos-Heredero, C. (2017). Customer relationship management (CRM) and BIG DATA: A conceptual approach and their impact over the power of data applied to selling strategies, DYNA, Vol. 92, No. 3, 274-279, doi:10.6036/8071.

[12] Kaya Keleș, M. (2017). An overview: The impact of data mining applications on various sectors, Tehnicki Glasnik - Technical Journal, Vol. 11, No. 3, 128-132.

[13] Zhou, R., Liao, Y., Shen, W., Yang, S. (2020). Channel selection and fulfillment service contracts in the presence of asymmetric service information, International journal of production economics, Vol. 222, 107504, doi: 107504.1107504.17.

[14] Karraya, S., Siguéb, S.P. (2018). Offline retailers expanding online to compete with manufacturers: Strategies and channel power, Industrial Marketing Management, Vol. 71, 203-214, doi: 10.1016/j.indmarman.2018.01.004.

[15] Shi, Y., Zhou, L., Qu, T., Qi, Q. (2019). Introducing the marketplace or not? Risk analysis of channel strategy for online retailers, Industrial Management \& Data Systems, Vol. 120, No. 2, 366-387, doi: 10.1108/IMDS-03-20190183.

[16] Nie, J., Zhong, L., Yan, H., Yang, W. (2019). Retailers' distribution channel strategies with cross-channel effect in a competitive market, International Journal of Production Economics, Vol. 213, 32-45, doi: 10.1016/j.ijpe.2018.12. $\underline{019}$.

[17] Wang, K., Goldfarb, A. (2017). Can offline stores drive online sales?, Journal of Marketing Research, Vol. 54, No. 5, 706-719, doi: 10.1509/jmr.14.0518.

[18] Huang, J.-S., Pan, S.L., Liu, J. (2017). Boundary permeability and online-offline hybrid organization: A case study of Suning, China, Information \& Management, Vol. 54, No. 3, 304-316, doi: 10.1016/j.im.2016.08.002.

[19] Jayaswal, S., Jewkes, E.M. (2016). Price and lead time differentiation, capacity strategy and market competition, International Journal of Production Research, Vol. 54, No. 9, 2791-2806, doi: 10.1080/00207543.2016.1145816.

[20] Pekgün, P., Griffin, P.M., Keskinocak, P. (2016). Centralized vs. decentralized competition for price and lead-time sensitive demand. Decision Sciences, Forthcoming, Vol. 48, No. 6, 1198-1227.

[21] Li, Y.-B., Lin, C.-P., Wu, T.-J., Chen, J.-L. (2017). The study on price and lead time decisions in retailer-dominated dual-channel supply chain, Journal of Interdisciplinary Mathematics, Vol. 20, No. 3, 805-819, doi: 10.1080/ 09720502.2017.1358869.

[22] Zhao, S., Wu, F., Jia, T., Shu, L. (2018). The impact of product returns on price and delivery time competition in online retailing, Computers \& Industrial Engineering, Vol. 125, 658-667, doi:10.1016/j.cie.2018.01.007.

[23] Ye, F., Xie, Z., Tong, Y., Li, Y. (2020). Promised delivery time: Implications for retailer's optimal sales channel strategy, Computers \& Industrial Engineering, Vol. 144, 106474, doi:10.1016/i.cie.2020.106474

[24] Yang, J.Q., Zhang, X.M., Fu, H.Y., Liu, C. (2017). Inventory competition in a dual-channel supply chain with delivery lead time consideration, Applied Mathematical Modelling, Vol. 42, 675-692, doi:10.1016/j.apm.2016.10.050.

[25] He, L., Zhang, X., Wang, Q.P., Hu, C.L. (2018). Game theoretic analysis of supply chain based on mean-variance approach under cap-and-trade policy, Advances in Production Engineering \& Management, Vol. 13, No. 3, 333344, doi: 10.14743/apem2018.3.294.

[26] Chiang, W.K., Chhajed, D., Hess, J.D. (2003). Direct marketing, indirect profits: A strategic analysis of dual-channel supply-chain design, Management Science, Vol. 49 , No. 1, 1-20, doi: 10.1287/mnsc.49.1.1.12749.

[27] Balcázar-Camacho, D.A., López-Bello, C.A., Adarme-Jaimes, W. (2016). Strategic guidelines for supply chain coordination in healthcare and a mathematical model as a proposed mechanism for the measurement of coordination effects, DYNA, Vol. 83, No.197, 203-211, doi: 10.15446/dyna.v83n197.55596. 


\title{
Optimalna izbira prodajnega kanala trgovca v dvokanalni dobavni verigi glede na želeni dobavni rok potrošnika
}

\author{
Hu, Y.S. ${ }^{a, b}$, Zeng, L.H. ${ }^{a}$, Huang, Z.L. ${ }^{c}$, Cheng, Q. ${ }^{\text {, }}$ \\ aschool of Transportation, Fujian University of Technology, Fuzhou, P.R. China \\ bSchool of Business, Maynooth University, Maynooth, County Kildare, Republic of Ireland \\ 'Research Center of Logistics, Fuzhou University, Fuzhou, P.R. China
}

\begin{abstract}
POVZETEK
Raziskava se ukvarja z vprašanjem, kako naj trgovci vzpostavijo prodajne kanale, da bi obdržali potrošnike, v konkurenci z neposredno spletno prodajo proizvajalcev blaga. Empirične raziskave so pokazale, da je dobavni rok ključni dejavnik pri izbiri spletnih kanalov. Da bi analizirali vpliv želja potrošnikov glede dobavnega roka na izbiro kanalov in na cenovno strategijo trgovcev, je bila funkcija želenega dobavnega roka spremenjena iz linearne v eksponentno. Na podlagi funkcije želenega dobavnega roka je bilo ocenjeno povpraševanje potrošnikov v okviru mešane dvokanalne dobavne verige, v kateri sodelujeta proizvajalec in trgovec. Nato so bili vzpostavljeni in rešeni modeli igre Stackelberg v okviru različnih prodajnih strategij trgovca. Rezultati kažejo, da ima želja potrošnikov glede dobavnega roka štiri posledice na prodajno strategijo trgovcev. Prvič, strategija dvokanalne prodaje je optimalna izbira za trgovce. V primeru spletne prodaje in dvokanalne prodaje se za trgovca stopnja dobička poveča, ko se poveča koeficient želenega dobavnega roka. Drugič, za trgovca je optimalna cena blaga v primeru samo spletne in samo trgovinske prodaje pozitivno povezana s koeficientom želenega dobavnega roka. Nasprotno pa je za proizvajalca optimalna cena blaga $v$ primeru samo spletne prodaje negativno povezana s koeficientom želenega dobavnega roka. Tretjič, za trgovca je optimalna cena blaga v primeru spletne prodaje višja od optimalne cene blaga $\mathrm{v}$ primeru trgovinske prodaje in višja od cene neposredne spletne prodaje proizvajalca blaga. Četrtič, da bi trgovec in proizvajalec rešila spor, ki ga povzroča optimalna izbira trgovca, lahko sprejmeta pogodbo, ki temelji na nadomestilu. Ta študija v odločanje o prodajnih kanalih uvaja želeni dobavni rok in zagotavlja teoretično podlago za gradnjo mešanih prodajnih kanalov trgovca v praksi.
\end{abstract}

\section{PODATKI O ČLANKU}

Ključne besede:

Dobavna veriga;

Dvokanalna prodaja;

Želja potrošnika;

Želeni dobavni rok;

Izbira kanala;

Usklajevanje kanalov

*Kontaktna oseba:

chengquan@fzu.edu.cn

(Cheng, Q.)

Zgodovina članka:

Prejet 20. septembra 2020

Popravljen 6. decembra 2020

Sprejet 9. decembra 2020 